Supplemental materials for

\title{
Bacterial production of pinene by a laboratory-evolved
} pinene-synthase

Miki Tashiro, Hiroshi Kiyota, Shigeko Kawai-Noma, Kyoichi Saito, Masahiko Ikeuchi, Yoko lijima,

Daisuke Umeno 
SI-1 Establishment of the screening condition for PS mutants with elevated cellular activity.

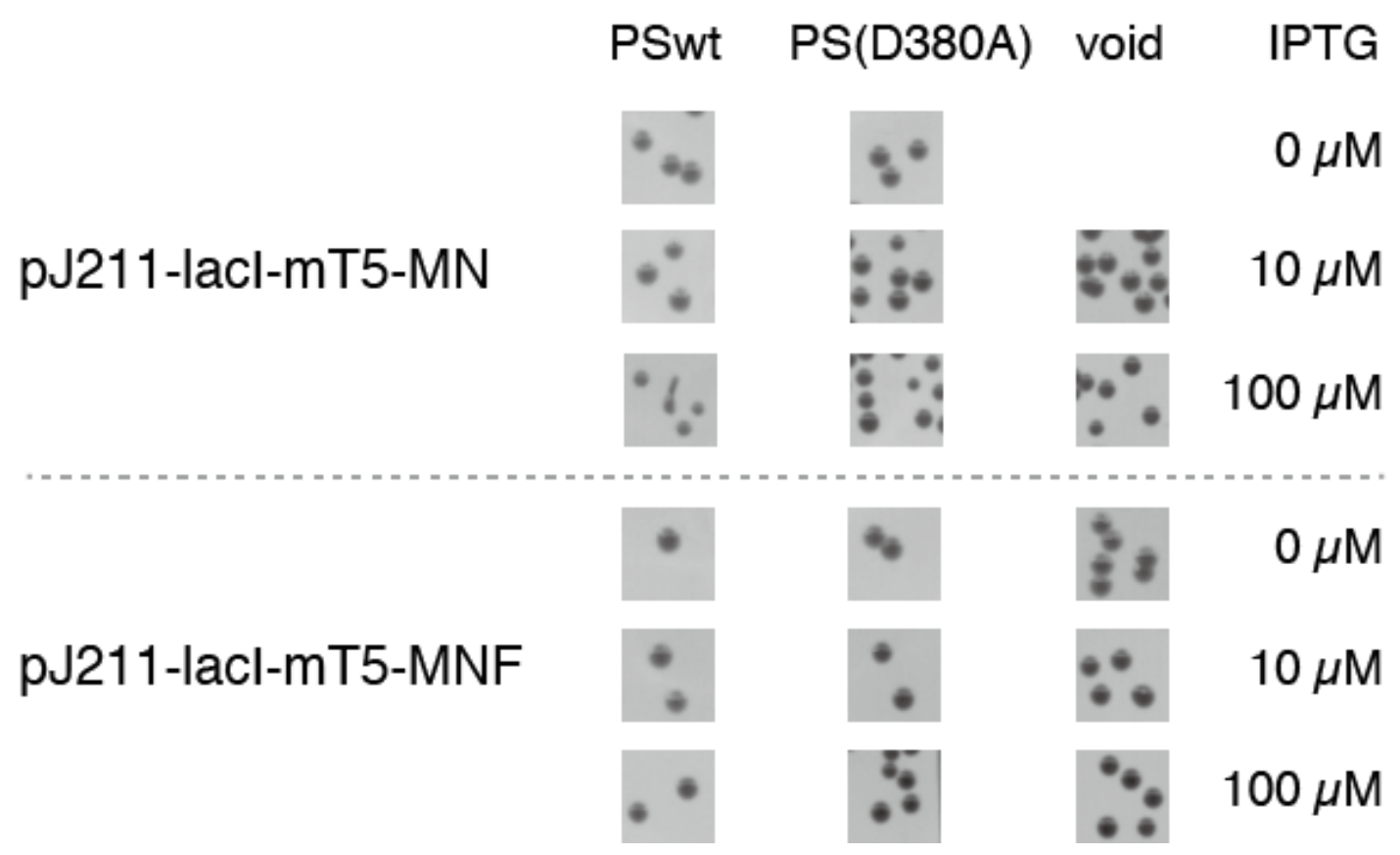

Fig. S1. Effect of expressing active (wild-type) and inactive PS on the colony hue of the XL1-Blue containing pJ211-lacl-mP ${ }_{T 5}-\mathrm{MN}$ or pJ211-lacl-mP $\mathrm{P}_{\mathrm{T5}}-\mathrm{MNF}$. 


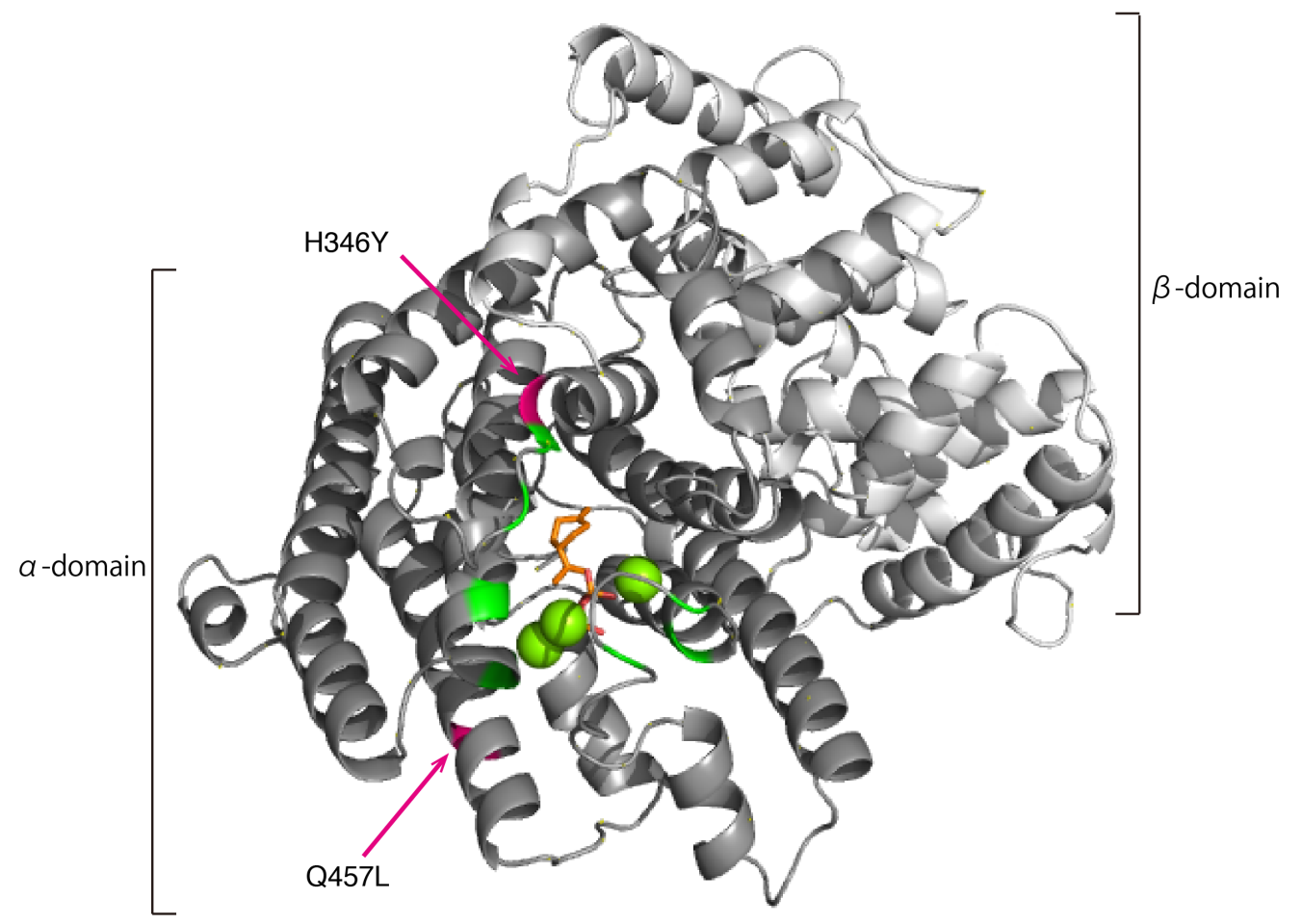

Fig. S2. Structural mapping of the mutations found in PSmut The PS structure was modeled using the SWISS-MODEL server ${ }^{1}$ based on the crystal structure of a monoterpene synthase (PDB: $1 \mathrm{~N} 22^{2}$ ). The structure of a substrate analog (orange) and manganese (green sphere) were taken from the crystal structure of $1 N 22$. The conserved residues (RXR, DDXXD, NSE/DTE) in the catalytic core and the mutations found in PSmut are shown in green and pink, respectively. 


\section{SI-3 E. coli production of pinene using PS-GPPS fusion.}

\section{Background:}

In some cases, the cellular production of mono-TPS is significantly elevated by fusion with the precursor supplier enzyme GPPS, likely due to (partial) substrate channeling. Sarria et al. systematically tested nine different fusions of GPPS and PSs from three conifer species (Abies grandis, Piece abies, and Pinus taeda) ${ }^{3}$. Five of the nine fusion constructs exhibited higher performance in the cellular context. The cells expressing these constructs accumulated more pinene than those co-expressing these enzymes as independent translational units. Following this success, we fused AgGPPS and PSmut with two different orders, yielding GPPS-PS and PS-GPPS.

\section{Plasmid construction:}

The plasmids pJ404-PS:GPPS his $_{\text {and pJ404-PSmut:GPPS }}$ his $($ Fig. S3a) were constructed as follows: The vector fragments were amplified by PCR using appropriate primers (pJ404-PtPS-GPPS2fus vector-F and pJ404-PtPS-GPPS2fus vector-R; for primer sequences, see Table S1). The fragment of gpps was PCR-amplified (using primers pJ404-PtPS-GPPS2fusion-ins-F and pJ404-PtPS-GPPS2fusion-ins-R) from pJ404-PS his $_{\text {-GPPS. }}$ These fragments were ligated by Golden Gate cloning ${ }^{4}$, yielding pJ404-PS:GPPS ${ }_{\text {his }}$ and pJ404-PSmut:GPPS his. $_{\text {. }}$

The plasmids pJ404-GPPS:PS ${ }_{\text {his }}$ and pJ404-GPPS:PSmut were constructed as follows. The PS fragment was PCR-amplified with a different primer set (pJ404-GPPS:PtPS-PF and PtPS-SLIC-R1) from pJ404-PSwt. The fragment of gpps was amplified by PCR using primers pJ404-RBS-GPPS:PtPS-F1 and pJ404-GPPS:PtPS-GR from pJ404-PS his -GPPS. The resultant fragments coding for $P S$ and GPPS were fused by soeing $P C R{ }^{5}$ using the primers pJ404-RBS-GPPS:PtPS-F2 and pJ404-GPPS:PtPS-GR. The resulting fragments were subcloned into the Xbal / HindIII site of pJ404-PS his $_{\text {or }}$ pJ404-PSmut $t_{\text {is }}$ using SLIC ${ }^{6}$.

\section{E. coli production of pinene using fusion proteins:}

The plasmids expressing fusion proteins were transformed into $E$. coli containing XL1-Blue containing pMev-idi, cutured, harvested, and compared in terms of pinene accumulation, following the protocol described for Fig. 5 (Fig. S3).

Neither of the constructs (GPPS-PS and PS-GPPS) exhibited higher performance in pinene production than the construct expressing GPPS and PSs in different translational frames. Fusion of GGPS to the N-terminus of PSs resulted in a drastic decrease in their functions. In contrast, the fusion of GGPS to the C-terminus of PSs resulted in a similar or slightly lower production of pinene with co-expressed GGPS and PS. This observation contradicts findings previously reported for $A$. grandis PS ${ }^{3}$. Sarria et al. showed that the fusion of GPPS to its N-terminus (GPPS-PS type) resulted in a $35 \%$ increase in pinene production, whereas the fusion of GPPS to its C-terminus (PS-GPPS type) dramatically reduced cellular activity. PSmut showed higher performance than PSwt in both contexts. 
a

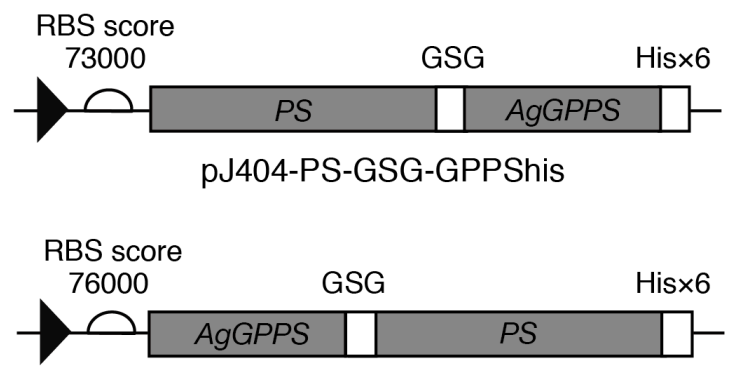

pJ404-GPPS-GSG-PShis b

Pinene production (mg/L-culture)

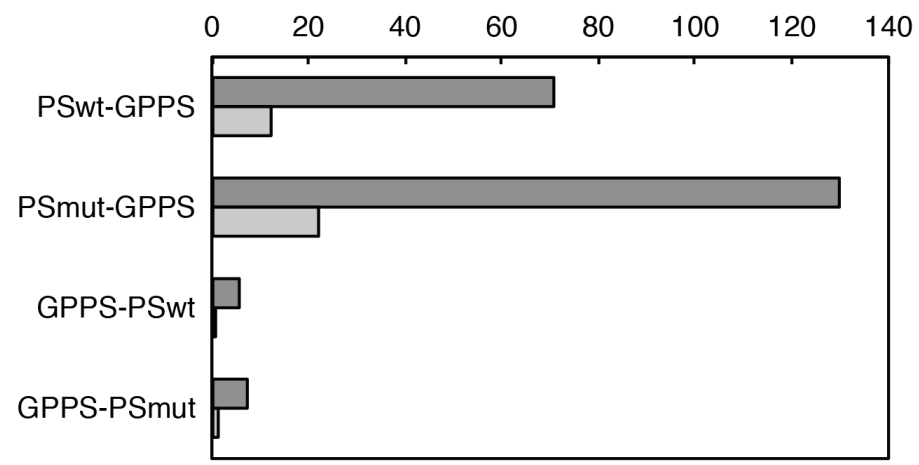

Fig. S3. Production of pinenes by E. coli expressing the fusions of AgGPPS and PSs.

(a) Plasmid construct for expression of the fusion of PSs and GPPS. (b) Pinene production by the fusion of PSs and GPPS. The results are shown as the average of two biological replicates. Each pair of replicates produced similar amounts of pinenes. 


\section{SI-4 Sequences of pJ211-lacl-mP ${ }_{T 5}-M N F$ and pJ404-PSwt}

pJ211-lacl-mP ${ }_{\text {T5 }}$-MNF

GCTTTTAGAAAAACTCATCGAGCATCAAATGAAACTGCAATTTATTCATATCAGGATTATCAATACCATATT TTTGAAAAAGCCGTTTCTGTAATGAAGGAGAAAACTCACCGAGGCAGTTCCATAGGATGGCAAGATCCTGGT ATCGGTCTGCGATTCCGACTCGTCCAACATCAATACAACCTATTAATTTCCCCTCGTCAAAAATAAGGTTAT CAAGTGAGAAATCACCATGAGTGACGACTGAATCCGGTGAGAATGGCAAAAGTTTATGCATTTCTTTCCAGA CTTGTTCAACAGGCCAGCCATTACGCTCGTCATCAAAATCACTCGCATCAACCAAACCGTTATTCATTCGTG ATTGCGCCTGAGCGAGGCGAAATACGCGATCGCTGTTAAAAGGACAATTACAAACAGGAATCGAGTGCAACC GGCGCAGGAACACTGCCAGCGCATCAACAATATTTTCACCTGAATCAGGATATTCTTCTAATACCTGGAACG CTGTTTTTCCGGGGATCGCAGTGGTGAGTAACCATGCATCATCAGGAGTACGGATAAAATGCTTGATGGTCG GAAGTGGCATAAATTCCGTCAGCCAGTTTAGTCTGACCATCTCATCTGTAACATCATTGGCAACGCTACCTT TGCCATGTTTCAGAAACAACTCTGGCGCATCGGGCTTCCCATACAAGCGATAGATTGTCGCACCTGATTGCC CGACATTATCGCGAGCCCATTTATACCCATATAAATCAGCATCCATGTTGGAATTTAATCGCGGCCTCGACG TTTCCCGTTGAATATGGCTCATATTCTTCCTTTTTCAATATTATTGAAGCATTTATCAGGGTTATTGTCTCA TGAGCGGATACATATTTGAATGTATTTAGAAAAATAAACAAATAGGGGTCAGTGTTACAACCAATTAACCAA TTCTGAACATTATCGCGAGCCCATTTATACCTGAATATGGCTCATAACACCCCTTGTTTGCCTGGCGGCAGT AGCGCGGTGGTCCCACCTGACCCCATGCCGAACTCAGAAGTGAAACGCCGTAGCGCCGATGGTAGTGTGGGG ACTCCCCATGCGAGAGTAGGGAACTGCCAGGCATCAAATAAAACGAAAGGCTCAGTCGAAAGACTGGGCCTT TCGCCCGGGCTGCAGTTAATGGTCGCGGGCGGCGACCAGTTCGCAAATATAGGCGAGCGCGGCGCCGTCAAC GTCAGCGTTCCGTAAATGGCGCTGCGCCGCCTCGATATGGAACGCCAACTTTTCCTTCGCGCCGGCAAGCGA CAGCAACGCTGGATACGTCGCTTTGTTGTTGCTTTGGTCGCTGCCGACCGGCTTGCCGATTTTTTCTTCTGC СCCTTCAATATCGAGAATATCATCGCGAATTTGAAAGGCAAGGCCTAGATGGGCGGCGAATTCGTCAAGCTC CCGCGTTTGCCGGGCATCAGCGCCGCCGATCAAGGCGCCGGCGTGCACGCTGTATTGCAGCATTTTCCCGGT TTTATGCCGATGAATGTATTCGAGCTCCGAAAGCGTCAGCGTTTTCCCCTCTCCTTCCATATCGGCTGCCTG ACCGGCGACCATCCCTTCCGGACCGGCCGCTTTCGCCAGCCGTTCGATGAGCCGAAGCCGGACGGAAGGAGG GATGCGCTCATCGTCGATTTCGGTGATCAATTGAAACGCGTACGTCAACAACCCGTCCCCCGCCAAGATGGC CATCGCCTCGCCGAACACTTTATGGTTCGTCGGCTTGCCGCGCCGCAAATCATCGTTGTCCATGCTCGGCAA ATCATCATGGATCAAAGAGTACGTATGGATCATTTCAATCGCGCAGGCGACGGGCAATCCGACCGCCGGGTC TTTGCCGAGCGCCCGAACGGTGGACAGAAGCAGCAACGGACGGATTCGTTTGCCGCCGGCCTCCAATGAGTA CGCCATCGCCTTTTTCAGCTTCGCCGGCCCTTCTAAGCGCTCTATATAACGGGAGAGCGCTGTTTCCACCGC CTGTTTTTGCTCGTTGAGAAACTGTTCAACTGAAAGCTGCGCCATCGCTTACTCCTCCTGGGCTAATTAGGG GGTGTCGCCCTTGAAGTCGACTCACTGCCCGCTTTCCAGTCGGGAAACCTGTCGTGCCAGCTGCATTAATGA ATCGGCCAACGCGCGGGGAGAGGCGGTTTGCGTATTGGGCGCCAGGGTGGTTTTTCTTTTCACCAGTGAGAC TGGCAACAGCTGATTGCCCTTCACCGCCTGGCCCTGAGAGAGTTGCAGCAAGCGGTCCACGCTGGTTTGCCC CAGCAGGCGAAAATCCTGTTTGATGGTGGTTAACGGCGGGATATAACATGAGCTATCTTCGGTATCGTCGTA TCCCACTACCGAGATATCCGCACCAACGCGCAGCCCGGACTCGGTAATGGCGCGCATTGCGCCCAGCGCCAT CTGATCGTTGGCAACCAGCATCGCAGTGGGAACGATGCCCTCATTCAGCATTTGCATGGTTTGTTGAAAACC GGACATGGCACTCCAGTCGCCTTCCCGTTCCGCTATCGGCTGAATTTGATTGCGAGTGAGATATTTATGCCA GCCAGCCAGACGCAGACGCGCCGAGACAGAACTTAATGGGCCCGCTAACAGCGCGATTTGCTGGTGACCCAA TGCGACCAGATGCTCCACGCCCAGTCGCGTACCGTCCTCATGGGAGAAAATAATACTGTTGATGGGTGTCTG GTCAGAGACATCAAGAAATAACGCCGGAACATTAGTGCAGGCAGCTTCCACAGCAATGGCATCCTGGTCATC 
CAGCGGATAGTTAATGATCAGCCCACTGACGCGTTGCGCGAGAAGATTGTGCACCGCCGCTTTACAGGCTTC GACGCCGCTTCGTTCTACCATCGACACCACCACGCTGGCACCCAGTTGATCGGCGCGAGATTTAATCGCCGC GACAATTTGCGACGGCGCGTGCAGGGCCAGACTGGAGGTGGCAACGCCAATCAGCAACGACTGTTTGCCCGC CAGTTGTTGTGCCACGCGGTTGGGAATGTAATTCAGCTCCGCCATCGCCGCTTCCACTTTTTCCCGCGTTTT CGCAGAAACGTGGCTGGCCTGGTTCACCACGCGGGAAACGGTCTGATAAGAGACACCGGCATACTCTGCGAC ATCGTATAACGTTACTGGTTTCATATTCACCACCCTGAATTGACTCTCTTCCGGGCGCTATCATGCCATACC GCGAAAGGTTTTGCGCCACCATGGGCATTTAGAATAAATTTTGTGTCGCCCTTAATTGTGAGCGGATAACAA TTACGAGCTTCATGCACAGTGAAATCATGAAAAATTTATGTGCAATGTGAGCGGATAACAATTATAATATGT GGAATTGTGAGCGCTCACAATTCCACAACGGTTTCCCTCTAGGAATTCCTAGATTCTAGATAGATAATCTCC ССTCTCGCCAAGGCCGCAACAGCATGACTATGATGAATATGAACTTTAAGTACTGCCATAAGATTATGAAGA AACACAGCAAATCTTTCAGCTACGCGTTCGATCTGTTGCCTGAGGACCAACGCAAAGCGGTTTGGGCGATCT ATGCGGTCTGCCGCAAAATTGATGACAGCATCGACGTTTATGGCGATATCCAGTTCCTGAATCAAATCAAAG AGGATATTCAAAGCATCGAGAAGTACCCGTACGAGCATCACCACTTCCAATCTGATCGCCGCATTATGATGG CGCTGCAACACGTGGCGCAGCACAAGAACATTGCGTTTCAGAGCTTTTACAACCTGATCGACACCGTGTATA AAGACCAGCACTTTACTATGTTTGAAACCGACGCGGAGCTGTTTGGCTATTGTTACGGTGTCGCAGGCACGG TTGGCGAGGTTCTGACCCCGATCCTGTCCGACCATGAAACCCACCAGACTTATGACGTCGCGCGTCGTCTGG GCGAGAGCCTGCAACTGATCAACATCCTGCGTGATGTAGGCGAAGATTTTGATAATGAGCGTATTTACTTCT CCAAGCAGCGCCTGAAGCAATACGAGGTCGATATCGCGGAAGTCTACCAGAACGGTGTGAATAATCATTACA TTGACCTGTGGGAGTATTACGCAGCGATTGCTGAGAAAGATTTCCAAGATGTAATGGACCAGATTAAGGTGT TTAGCATTGAGGCGCAACCGATTATTGAGCTGGCCGCTCGTATCTACATCGAGATTTTGGATGAGGTGCGAA GCGAACTATACCCTGCATGAACGCGTCTTTGTGGACAAACGCAAAAAGGCAAAACTGTTCCACGAGATCAAC AGCAAATACCATCGTATCTAACTCGAGAACTAACCGCTCAAAATAAGGGGTTATTATAATGAAAATTGCCGT AATTGGTGCTGGTGTAACGGGTTTGGCTGCTGCTGCACGTATTGCTAGCCAGGGTCACGAAGTGACCATTTT TGAGAAGAATAACAACGTTGGCGGTCGTATGAATCAACTGAAGAAGGATGGTTTCACCTTCGACATGGGTCC AACGATTGTTATGATGCCGGATGTTTACAAAGACGTTTTCACGGCATGTGGCAAGAATTATGAGGACTACAT TGAGCTGCGCCAATTGCGTTACATCTATGATGTGTACTTCGACCATGACGATCGCATCACGGTGCCGACCGA TCTGGCCGAATTGCAGCAGATGCTGGAGAGCATTGAACCGGGCAGCACCCACGGTTTCATGAGCTTCTTGAC CGACGTCTATAAGAAGTATGAGATTGCACGTCGTTATTTCCTGGAACGCACGTACCGTAAGCCGAGCGACTT CTACAATATGACCAGCCTGGTTCAGGGCGCGAAGCTGAAAACCCTGAATCATGCGGATCAACTGATTGAACA TTACATCGACAATGAGAAAATCCAGAAGCTGCTGGCATTTCAGACGCTGTACATTGGTATCGACCCTAAACG TGGTCCGTCCCTGTACTCTATTATCCCGATGATCGAAATGATGTTTGGCGTTCACTTTATCAAGGGTGGTAT GTACGGTATGGCCCAGGGTCTGGCCCAGCTGAATAAAGATCTGGGCGTGAACATCGAGTTGAACGCGGAAAT TGAGCAGATCATTATTGACCCGAAATTCAAGCGTGCCGACGCTATCAAGGTTAACGGTGACATTCGTAAATT CGACAAAATCCTGTGCACCGCCGATTTTCCGTCTGTGGCCGAGTCCCTGATGCCAGATTTTGCGCCGATTAA GAAATATCCGCCGCACAAGATCGCGGATTTGGACTATAGCTGTAGCGCATTTCTGATGTATATCGGTATTGA CATTGACGTCACCGATCAGGTGCGTCTGCACAATGTGATCTTCAGCGACGACTTTCGTGGCAATATCGAAGA AATCTTCGAGGGTCGCTTGTCGTACGATCCGTCGATTTATGTGTACGTTCCGGCAGTCGCGGACAAAAGCCT GGCACCTGAGGGCAAGACCGGTATTTACGTTCTGATGCCTACGCCGGAACTGAAAACGGGTTCGGGTATTGA TTGGTCTGATGAAGCGTTGACGCAGCAGATCAAAGAAATCATTTATCGCAAGTTGGCGACCATCGAAGTTTT CGAGGACATTAAGTCCCACATCGTCAGCGAAACCATCTTCACCCCGAATGATTTCGAACAAACGTATCACGC TAAGTTCGGCAGCGCGTTTGGCCTGATGCCGACCCTGGCCCAGAGCAACTATTACCGTCCACAAAACGTTAG CCGTGACTACAAAGACCTGTACTTTGCGGGTGCGAGCACCCATCCGGGTGCGGGCGTTCCGATCGTCCTGAC GAGCGCGAAAATCACCGTGGACGAAATGATTAAAGATATTGAGCGTGGTGTCTAAGGGCCCCTAGATACTAG 
TCGTCAAAAGGGCGACACAAAAGGTATTCTAAATGCATAATAAATACTGATAACATCTTATAGTTTGTATTA TATTTTGTATTATCGTTGACATGTATAATTTTGATATCAAAAACTGATTTTCCCTTTATTATTTTCGAGATT TATTTTCTTAATTCTCTTTAACAAACTAGAAATATTGTATATACAAAAAATCATAAATAATAGATGAATAGT TTAATTATAGGTGTTCATCAATCGAAAAAGCAACGTATCTTATTTAAAGTGCGTTGCTTTTTTCTCATTTAT AAGGTTAAATAATTCTCATATATCAAGCAAAGTGACAGGCGCCCTTAAATATTCTGACAAATGCTCTTTCCC TAAACTCCCCCCATAAAAAAACCCGCCGAAGCGGGTTTTTACGTTATTTGCGGATTAACGATTACTCGTTAT CAGAACCGCCCAGGATGCCTGGCAGTTCCCTACTCTCGCCGCTGCGCTCGGTCGTTCGGCTGCGGGACCTCA GCGCTAGCGGAGTGTATACTGGCTTACTATGTTGGCACTGATGAGGGTGTCAGTGAAGTGCTTCATGTGGCA GGAGAAAAAAGGCTGCACCGGTGCGTCAGCAGAATATGTGATACAGGATATATTCCGCTTCCTCGCTCACTG ACTCGCTACGCTCGGTCGTTCGACTGCGGCGAGCGGAAATGGCTTACGAACGGGGCGGAGATTTCCTGGAAG ATGCCAGGAAGATACTTAACAGGGAAGTGAGAGGGCCGCGGCAAAGCCGTTTTTCCATAGGCTCCGCCCCCC TGACAAGCATCACGAAATCTGACGCTCAAATCAGTGGTGGCGAAACCCGACAGGACTATAAAGATACCAGGC GTTTCCCCCTGGCGGCTCCCTCGTGCGCTCTCCTGTTCCTGCCTTTCGGTTTACCGGTGTCATTCCGCTGTT ATGGCCGCGTTTGTCTCATTCCACGCCTGACACTCAGTTCCGGGTAGGCAGTTCGCTCCAAGCTGGACTGTA TGCACGAACCCCCCGTTCAGTCCGACCGCTGCGCCTTATCCGGTAACTATCGTCTTGAGTCCAACCCGGAAA GACATGCAAAAGCACCACTGGCAGCAGCCACTGGTAATTGATTTAGAGGAGTTAGTCTTGAAGTCATGCGCC GGTTAAGGCTAAACTGAAAGGACAAGTTTTGGTGACTGCGCTCCTCCAAGCCAGTTACCTCGGTTCAAAGAG TTGGTAGCTCAGAGAACCTTCGAAAAACCGCCCTGCAAGGCGGTTTTTTCGTTTTCAGAGCAAGAGATTACG CGCAGACCAAAACGATCTCAAGAAGATCATCTTATTAA

pJ404-PSwt (Xbal - Xhol)

TCTAGAAATAATTTTGTTTAACTTTTAGGAGGTAAAACATATGCGTAGACGTGGCGATTTTCATTCTAACCT GTGGGACGACGACTTAATCCAGAGCCTGAGCAGCCCGTATGGTGAGCCATCCTATCGCGAGCGTGCGGAGCG CCTGATTGGTGAGGTGAAGAACTCTTTCAACAGCATGAGCAATGAGGACGGTGAGAGCATCACCCCGCTGGA TGATCTGATTCAACGCTTGTGGATGGTTGACAGCGTGGAGCGTCTGGGTATTGACCGCCATTTCAAGAAAGA AATCAAGTCTGCACTGGATCACGTTTATCGCTACTGGAGCGAAAAGGGTATCGGCTGCGGCCGTGAGAGCGT GGTCACCGATCTGAATAGCACCGCACTGGGTCTGCGTACGTTGCGCCTGCACGGTTATGATGTGAGCGCTGA CGTGCTGAATCACTTCAAGAATCAAAGCGGCCAGTTCGCGTGCACCCTGAAACAGACCGAAGATCAGATCCG TACCGTTTTGAACCTGTACCGCGCGAGCCTGATCGCCTTCCCGGGTGAGAAAGTCATGGATGAAGCGGAGAG СTTCTCCGCAAAATACCTGAAAGAGGCCCTGCAAAAGATTCCGGTGAGCAGCTTTAGCCGTGAAATCGGTGA CGTCCTGGAGTACGGTTGGCACACGTATCTGCCGCGTCTGGAAGCGCGTAACTATATCGACGTGTTCGGTCA GGACACCGAGAACTCCAAGAGCTATATGAAAACGGAAAAGCTGCTGGAGCTGGCGAAGCTTGAGTTCAATAT CTTCCATGCGTTGCAGAAACGCGAGTTGGAGTACTTGGTTCGTTGGTGGAAAGGTTCGGGCTCCCCTCAGAT GACCTTCTGTCGTCATCGTCACGTTGAGTATTACACCCTGGCTAGCTGCATTGCGTTTGAACCTCAACACTC TGGCTTTCGTCTGGGTTTTGCCAAAGCCTGTCATATCATCACTGTCCTGGATGATATGTACGATACGTTTGG CACCCTTGACGAACTGGAGCTGTTTACCAGCGCTATTAAGCGCTGGGACCCGAGCGCCACGGAGTGCCTGCC GGAGTACATGAAGGGTGTTTACATGATTGTCTACAATACCGTGAATGAGATGAGCCAAGAAGCAGACAAAGC GCAAGGCCGCGATACCCTGAACTATTGCCGTCAGGCGTGGGAAGAGTATATTGACGCTTACATGCAAGAAGC CAAGTGGATTGCGTCGGGTGAAGTCCCAACCTTTGAAGAGTACTACGAAAATGGTAAGGTTTCCAGCGGTCA CCGTGTTAGCGCACTGCAACCGATCCTGACGACTGACATCCCGTTCCCGGAGCACGTCCTGAAAGAAGTGGA CATTCCGAGCCAACTGAATGACCTGGCAAGCGCGATTCTGCGTTTGCGTGGTGACACGCGTTGCTATCAGGC AGACCGCGCTCGTGGCGAAGAAGCAAGCTGTATTTCCTGTTACATGAAAGATAACCCGGGCACGACGGAAGA AGATGCGCTGAACCATCTGAACGCCATGATCTCCGATGTGATTAAAGGCCTGAACTGGGAACTGTTGAAGCC 
GAATAGCTCTGTCCCGATTAGCGCGAAAAAGCATGCATTCGATATTAGCCGTGCGTTTCACTGTGGCTACAA GTATCGCGACGGTTACAGCGTTGCGAACATCGAGACTAAATCACTGGTAAAACGTACCGTGATCGATCCGGT TACCTTGGGCAGCAGCCACCACCACCATCACCACTAATAACTCGAG 
Table S1. Primers used in this work.

\begin{tabular}{|c|c|c|}
\hline & Name & Sequence \\
\hline 1 & pJBEI-6409-mod & ctgctggtctctatcggtgtgt \\
\hline 2 & pJBEI-6409-mod-idi-R & $\begin{array}{l}\text { ggagatccttactcgagtttggatcctgcgagatccttatttaagc } \\
\text { tgggta }\end{array}$ \\
\hline 3 & pJBEI-6409-mod-GPPS-R & $\begin{array}{l}\text { ggagatccttactcgagtttggatccagatccttaattctgacgaa } \\
\text { atgccac }\end{array}$ \\
\hline 4 & pJ404-PtPS-GPPS2-F & $\begin{array}{l}\text { catcaccactaataactcgagttcttaaggaggtaatcccatggaa } \\
\text { tttgacttcaacaaatacatgga }\end{array}$ \\
\hline 5 & pJ404-PtPS-GPPS2-R & $\begin{array}{l}\text { gtcgcccttggggcgaattcttaattctgacgaaatgccacgtagt } \\
\text { ct }\end{array}$ \\
\hline 6 & pJ404-PtPS-IspA-F & $\begin{array}{l}\text { catcaccactaataactcgagacccacaggaggtataaccatggac } \\
\text { tttccgcagcaactcgaa }\end{array}$ \\
\hline 7 & pJ404-PtPS-IspA-R & $\begin{array}{l}\text { gtcgcccttggggcgaattcttatttattacgctggatgatgtagt } \\
\text { ccgct }\end{array}$ \\
\hline 8 & pJ211-T5(M)-F & gctcttcttatgtgcaatgtgagcggataacaattataatatgtgg \\
\hline 9 & pJ211-T5-R & gctcttctataaatttttcatgatttcactgtgcatgaagc \\
\hline 10 & FDSinsF & $\begin{array}{l}\text { acttcaagggcgacaccccctaattagcccaggaggagtaagcgat } \\
\text { ggc }\end{array}$ \\
\hline 11 & FDSinsR & $\begin{array}{l}\text { ggctcagtcgaaagactgggcctttcgccegggctgcagttaatgg } \\
\text { tcgcgggcgg }\end{array}$ \\
\hline 12 & (FDS)idi-insR & tcgttatactcccctgcagttaatggtcgcgggcgg \\
\hline 13 & FDS(idi)-insF & $\begin{array}{l}\text { gcgaccattaactgcaggggagtataacgaatgcaaacggaacacg } \\
\text { tcattttattg }\end{array}$ \\
\hline 14 & FDS(idi)-insR & $\begin{array}{l}\text { gctcagtcgaaagactgggcctttcgcccgggaatcgatttattta } \\
\text { agctgggtaaatgcagataatcgt }\end{array}$ \\
\hline 15 & pJ211-laclQ-F & ttttccatggtggtgcaaaacctttcgcggtatgg \\
\hline 16 & lacl-SLIC-to-pJ211MN-R & gtgtcgcccttgaagtcgactcactgcccgctttccagtc \\
\hline 17 & PtPS ADXXD-F & gcggatatgtacgatacgtttggcacc \\
\hline 18 & PtPS ADXXD-R & ccaaacgtatcgtacatatccgccaggacagtgatgatatgacag \\
\hline 19 & PtPS-Nhis-F & catcatcatcacagcggatcccgtagacgtggcgattttcattc \\
\hline 20 & PtPS-Nhis-R & gtgtcgcccttggggctcgagttacaaggtaaccggatcgatcacg \\
\hline 21 & PtPS-Chis-F & cttttaggaggtaaaacatatgcgtaga \\
\hline 22 & PtPS-Chis-R & tcgcccttggggctcgagttatta \\
\hline 23 & PtPS-H346Y-F & tttggtctct tcgttacgttgagtattacaccctggct \\
\hline 24 & PtPS-H346Y-R & tttggtctctacgatgacgacagaaggtcatct \\
\hline 25 & PtPS-Q457L-F & tttggtctctgaagccaagtggattgcgtc \\
\hline 26 & PtPS-Q457L-R & tttggtctctcttctagcatgtaagcgtcaatatactcttcc \\
\hline 27 & pJ404-PtPS-GPPS2fus vector-F & tttggtctctagccaccaccaccatcaccactaataa \\
\hline 28 & pJ404-PtPS-GPPS2fus vector-R & tttggtctctcaaggtaaccggatcgatcacgg \\
\hline 29 & pJ404-PtPS-GPPS2fusion-ins-F & $\begin{array}{l}\text { tttggtctctcttgggatccggtgaatttgacttcaacaaatacat } \\
\text { ggactccaa }\end{array}$ \\
\hline 30 & pJ404-PtPS-GPPS2fusion-ins-R & tttggtctctggctgctgccattctgacgaaatgccacgtagtctg \\
\hline 31 & PtPS-SLIC-R1 & aagcttcgccagctccagca \\
\hline 32 & pJ404-RBS-GPPS:PtPS-F2 & $\begin{array}{l}\text { gcgctcacaattccacaacggtttccctctagaacgegtcccagca } \\
\text { cc }\end{array}$ \\
\hline
\end{tabular}




\begin{tabular}{|l|l|l|}
\hline 33 & pJ404-RBS-GPPS:PtPS-F1 & $\begin{array}{l}\text { agaacgcgtcccagcaccaagtaaggaggtagcatatggaatttga } \\
\text { cttcaacaatacatggactccaa }\end{array}$ \\
\hline 34 & pJ404-GPPS:PtPS-GR & accggatccattctgacgaatgccacgtagtctg \\
\hline 35 & pJ404-GPPS:PtPS-PF & $\begin{array}{l}\text { tacgtggcatttcgtcagaatggatccggtcgtagacgtggcgatt } \\
\text { ttcattc }\end{array}$ \\
\hline 36 & pTHTH-vector-R & tttggtctctcatatgatccttatcgtcatcgtcgtaca \\
\hline 37 & pTHTH-vector-F & tttggtctctctagaggatctgttaacctcacattggattcttc \\
\hline 38 & pTHTH-PtPS ins F & tttggtctcttatgcgtagacgtggcgattttca \\
\hline 39 & pTHTH-PtPS ins R & tttggtctctctagattacaaggtaaccggatcgatcac \\
\hline 40 & pET15b-R-BamHI & TTTTGGATCCgctgtgatgatgatgatgatggc \\
\hline 41 & pET15b-F-Xhol & TTTTCTCGAGggctgctaacaaagcccgaaa \\
\hline
\end{tabular}

\section{References}

(1) Biasini, M., Bienert, S., Waterhouse, A., Arnold, K., Studer, G., Schmidt, T., Kiefer, F., Cassarino, T. G., Bertoni, M., Bordoli, L., and Schwede, T. (2014) SWISS-MODEL: modelling protein tertiary and quaternary structure using evolutionary information. Nucleic Acids Res. 42, W252-W258.

(2) Whittington, D. a, Wise, M. L., Urbansky, M., Coates, R. M., Croteau, R. B., and Christianson, D. W. (2002) Bornyl diphosphate synthase: structure and strategy for carbocation manipulation by a terpenoid cyclase. Proc. Natl. Acad. Sci. U. S. A. 99, 15375-80.

(3) Sarria, S., Wong, B., Martín, H. G., Keasling, J. D., and Peralta-Yahya, P. (2014) Microbial Synthesis of Pinene. ACS Synth. Biol. 3, 466-475.

(4) Bryksin, A. V, and Matsumura, I. (2010) Overlap extension PCR cloning: a simple and reliable way to create recombinant plasmids. Biotechniques 48, 463-5.

(5) Horton, R. M., Cai, Z. L., Ho, S. N., and Pease, L. R. (1990) Gene splicing by overlap extension: tailor-made genes using the polymerase chain reaction. Biotechniques 8, 528-35.

(6) Stevenson, J., Krycer, J. R., Phan, L., and Brown, A. J. (2013) A Practical Comparison of Ligation-Independent Cloning Techniques. PLoS One 8, e83888. 